\title{
Técnicas da Pesquisa Operacional aplicadas a um Problema de Cobertura de Arcos
}

A. SMIDERLE, UFPR, Programa de Pós-Graduação em Métodos Numéricos em Engenharia e Faculdade Mater Dei, 85505-000 Pato Branco, PR, Brasil.

M.T. ARNS STEINER, V.E. WILHELM, UFPR, Departamento de Matemática e Programa de Programa de Pós-Graduação em Métodos Numéricos em Engenharia, CP: 19081, 81531-990 Curitiba, PR, Brasil.

\begin{abstract}
Resumo. Neste trabalho, é proposta uma metodologia para a obtenção de uma solução otimizada de um problema de cobertura de arcos utilizando algumas técnicas da Pesquisa Operacional. A metodologia aqui apresentada consta de duas fases. $\mathrm{Na} 1^{a}$ fase utiliza-se um algoritmo genético aplicado ao problema das $p$-medianas cuja resposta pode ser melhorada com a heurística clássica de Teitz e Bart. A partir da definição das medianas necessárias para o problema, determina-se os grupos (clusters) de pontos de demanda a serem designados a cada mediana através do algoritmo de Gillett e Johnson Adaptado. Na $2^{a}$ fase, a partir da definição dos clusters de atendimento, é feito o roteamento em cada cluster para ter-se a seqüência de pontos a serem transpassados, utilizando o modelo matemático do Problema Carteiro Chinês. A validação da metodologia foi obtida através da aplicação da mesma a um estudo de caso comparando-se a solução otimizada com a solução adotada por ocasião a coleta de dados.
\end{abstract}

\section{Introdução}

Serviços do setor público como, por exemplo, varredura de ruas, coleta de lixo, entrega de correspondências, inspeção de linhas de água, eletricidade ou gás, monitoramento de estacionamentos regulamentados são realizados a partir da utilização de recursos humanos em grande escala. Para a execução dos serviços mencionados é necessário haver a percorrida, através de caminhada (a pé) ou com algum tipo de veículo, ao longo dos trechos (ou arcos) produtivos da região em estudo. Por este motivo, estes problemas, os quais objetiva-se otimizar, são chamados de problemas de cobertura de arcos.

Nos problemas de roteamento, o Problema do Carteiro Chinês (PCC) caracteriza os problemas de cobertura de arcos, em que, sobre uma rede viária, deve-se percorrer todos os arcos exatamente uma única vez. O seu estudo é destacado por alguns pesquisadores como, por exemplo: Costa et al. [4] propõem uma solução para o problema de entrega de correspondências realizada pelos serviços postais, onde o Algoritmo do Carteiro Chinês é comparado com vários algoritmos de cobertura de nós; Stern e Dror [12] aplicam o Algoritmo do Carteiro Chinês no estudo 
das rotas dos leituristas de medidores elétricos, onde o resultado obtido apresentou uma redução de $40 \%$ do número de trabalhadores empregados, mostrando a aplicabilidade deste estudo.

Eglese e Murdock [6] apresentam um software para otimizar o serviço de limpeza de ruas com veículos vassoura. Este estudo foi aplicado no noroeste da Inglaterra, com a intenção de desenvolver uma rota a ser seguida por cada varredor cuja distância total percorrida fosse minimizada, respeitadas as restrições com relação à quantidade de trabalho e ao tempo hábil em cada dia. Ghiani e Improta [7] apresentam uma variante do clássico problema do Carteiro Chinês, o problema do Carteiro Chinês Hierárquico, cujos arcos são divididos em agrupamentos (clusters) e a relação precedente é definida nos clusters. Sua aplicação prática pode ser vista nos controles de neve e gelo nas ruas e estradas.

Já para os problemas de $p$-medianas, onde o objetivo é localizar em uma rede contendo $n$ nós, $p$ nós (denominados medianas), de forma a minimizar a soma das distâncias de cada nó até a sua mediana mais próxima, pode-se destacar os pesquisadores: Senne e Lorena [10] que apresentam testes computacionais que demonstram a eficiência de algoritmos para a localização de $p$-medianas, considerando problemas da literatura e problemas reais obtidos a partir de SIG (Sistemas de Informações Geográficas) e Corrêa et al. [3], que utilizam o problema das p-medianas para decidir sobre os melhores locais de provas para a realização do vestibular em uma universidade.

A metodologia proposta neste trabalho é apresentada através de sua aplicação no serviço de leitura dos medidores das contas de água, efetuado pela empresa de saneamento básico do município de Pato Branco, PR. Visando a melhoria do referido serviço, a metodologia aqui apresentada consta, basicamente, de duas fases: a) estabelecer a área de atendimento para cada leiturista, levando em consideração que a quantidade de faturas emitidas seja, aproximadamente, a mesma para todos eles; b) determinar o percurso a ser feito por cada leiturista dentro de sua área de atendimento (definida na fase anterior), adequando-o às características particulares do tipo de serviço.

Os serviços de saneamento básico oferecidos pela SANEPAR (Companhia de Saneamento do Paraná) são operacionalizados por leituristas (também chamados de agentes) que fazem a leitura dos dados, digitam os mesmos em um microcomputador, emitem a fatura e a entregam ao cliente; além disso, é função do leiturista, conferir dados do logradouro, prestar informações diversas, vender serviços como consertos dos mais diversos e verificar as condições de instalação da ligação predial de água (conjunto formado pelo ramal predial e o cavalete) e hidrômetro dos locais visitados. Por isso a necessidade de otimizar, ao máximo, o caminho de percorrida do leiturista, para que o tempo gasto com o seu deslocamento seja o menor possível.

\section{Descrição do Problema Real}

A SANEPAR, empresa estadual de economia mista, é responsável pelas ações de saneamento básico em quase todo o Estado do Paraná.

O saneamento básico do Paraná, no início da década de 60, possuía um índice 
de atendimento muito baixo. Apenas $8,3 \%$ da população era servida por rede de abastecimento de água, e apenas 4,1\% com rede de esgoto. Das 221 sedes municipais da época, 19 possuíam todos os serviços e 37 somente o de água. Das 20 cidades mais populosas do Estado, segundo o censo de 1950, apenas 11 tinham serviço de água. Hoje, $98 \%$ da população urbana é abastecida com água tratada; são mais de 7 milhões de habitantes distribuídos entre as 619 localidades atendidas pela Sanepar.

A SANEPAR unidade de Pato Branco, centraliza o atendimento de diversas cidades as quais totalizam 38.281 ligações de água. Após a distribuição da água, existe o trabalho de faturar os consumos. A empresa "Mercado" é uma empresa terceirizada que realiza esta atividade em Pato Branco, usando um sistema de processamento e emissão de fatura no próprio local do consumo. A utilização de microcomputadores portáteis reduz, em muito, os custos operacionais se comparada aos sistemas convencionais de faturamento (coleta das informações, deslocamento até a empresa para a digitação, consistência, processamento e a impressão da fatura, e retorno para o endereço para entregar a fatura ao cliente).

Por ocasião do levantamento dos dados, existiam na cidade de Pato Branco 14.097 ligações de água. Para a realização da leitura das contas de água, a cidade estava dividida, em 7 grupos. Para fins de estudo deste trabalho, foi considerado apenas um dos 7 grupos formado pelos bairros: Centro (parte), Parzianelo, Cadorin, Bancários, Pinheiros, Brasília, Vila Isabel, São Luiz, Bortot, Trevo da Guarani (parte). Este grupo foi escolhido por apresentar características de centro e bairros, ou seja, apresenta trechos curtos com muitos clientes e também trechos longos com poucos clientes.

O referido grupo era constituído por 2.932 ligações e estava dividido em 12 rotas para os leituristas. De acordo com as informações da empresa, eram percorridos 105.793 metros para efetuar a leitura destas 2.932 ligações, sendo que os 12 leituristas realizavam esse trabalho ao longo de um dia.

\section{Implementação Computacional e Resultados}

Preliminarmente à implementação computacional dos algoritmos, definiu-se a área de atuação dos leituristas na cidade de Pato Branco. Para fazer a leitura dos medidores de consumo de água, os leituristas devem percorrer, mensalmente, uma rede viária composta de ruas. Cada rua é dividida em trechos, onde cada trecho é uma face de quadra; desta forma, cada "pedaço" de rua, compreendido entre duas ruas transversais, contém 2 trechos, um para cada face de quadra. Os trechos podem ou não possuir medidores de água; os que possuem são chamados de trechos produtivos, os outros de improdutivos. Neste trabalho, em particular, todos os trechos são produtivos; desta forma o leiturista deverá passar por cada "pedaço" de rua, que contém 2 trechos produtivos, 2 vezes, atendendo aos 2 arcos.

O problema é determinar qual leiturista deverá atender a quais pontos de leitura, de maneira a percorrer seu trajeto de forma otimizada. Dessa forma, o problema a ser tratado, conforme já mencionado, foi dividido em duas fases: divisão da rede viária a ser estudada em clusters, sendo que cada cluster deverá ser percorrido por um leiturista e o roteamento do percurso (sequência de trechos a serem transpassados) nos diferentes clusters. 


\subsection{Etapas da Implementação Computacional}

Para a implementação computacional dos algoritmos, as duas fases mencionadas foram divididas em 4 etapas distintas, descritas detalhadamente em Smiderle [11].

$\mathrm{Na} 1^{a}$ etapa efetuou-se o cadastramento de 774 pontos no mapa digitalizado da cidade de Pato Branco que deverão ser atendidos por 12 leituristas. Esses pontos foram dispostos de maneira a mapear a parte da rede viária a ser estudada; eles foram introduzidos nas interseções das ruas (cruzamentos) e no meio de cada trecho produtivo. No mapa da cidade, pequenos símbolos indicam a presença destes pontos; assim sendo, um banco de dados é formado pelas coordenadas geográficas de cada um destes pontos.

$\mathrm{Na} 2^{a}$ etapa foi utilizado o Algoritmo Genético aplicado ao problema das $P_{-}$ Medianas (AGPMed), com $P=12$, para a determinação de 12 medianas, Barbosa [1] e Goldberg [8], e o Algoritmo de Teitz e Bart (T\&B) para o refinamento da resposta fornecida pelo AGPMed, Teitz \& Bart [13]. Para esta $2^{a}$ etapa algumas definições preliminares se fazem necessário, as quais são apresentadas a seguir.

Seja um grafo $G(V, A)$ não direcionado onde $V$ são os vértices e $A$ as arestas. Seja $v_{i}$ um vértice qualquer pertencente a $V$. Chama-se número de transmissão $(\sigma)$ à soma das menores distâncias existentes entre o vértice $v_{i}$ e todos os outros vértices do grafo. Sendo $n$ o número total de vértices do grafo, o número de transmissão é dado por:

$$
\sigma\left(v_{i}\right)=\sum_{j=1}^{n} w_{j} d\left(v_{i}, v_{j}\right), v_{i}, v_{j} \in V
$$

onde $d\left(v_{i}, v_{j}\right)$ é a menor distância entre $v_{i}$ e $v_{j}$ e $w_{j}$ é um peso associado ao vértice $v_{j}$. Assim, $v_{m}$ é uma mediana se, entre todos os vértices do grafo, é aquele que produz a menor soma total das distâncias desde si próprio até cada um dos vértices do grafo. Deste modo, forma-se um conjunto $V_{p}$, sendo que $V_{p} \subset V$, contendo um conjunto de $p$ vértices que é a solução ótima para o problema das p-medianas, ou seja, um conjunto para o qual o número de transmissão é mínimo.

Levando-se em conta estas informações, tem-se que para a aplicação do referido AGPMed, fixou-se o número máximo de iterações $k_{\text {máx }}=1.000$ e uma população variando de 75 a 200 indivíduos (cromossomos). O algoritmo pára quando atinge o número máximo de iterações ou quando a diferença dos valores de transmissão entre o melhor e o pior cromossomo é menor do que 2.000 metros. A seguir, descreve-se o procedimento básico deste algoritmo adotado neste trabalho.

\section{Algoritmo Genético para o problema das $P$-Medianas}

Passo 1. Construção da População Inicial:

- Gere uma lista $R=\left(r_{1}, \ldots, r_{m}\right)$, com $m$ cromossomos viáveis de $p$ elementos cada, sorteados entre os $v$ vértices do grafo;

- Calcule $C_{i}=f i$ iness $\left(r_{i}\right), r_{i} \in R$ e ordene $R$ tal que $C_{1} \leq \ldots \leq C_{m}$;

- Faça $k=0$ e defina o erro e o número máximo de iterações $k_{\text {máx }}$; 
Passo 2. Teste: Se $C_{m}-C_{1} \leq \varepsilon$ ou $k \geq k_{\text {máx }}$, então PARE e apresente $r_{1}$;

Passo 3. Seleção

- Selecione dois cromossomos, $r_{i}=\operatorname{Sel}(R)$ e $r_{j}=\operatorname{Sel}(R)$, com $r_{i} \neq r_{j}$

$$
\operatorname{Sel}(R)=\left\{r_{j} \in R \mid j=m+1-\left[\frac{-1+\sqrt{\left.1+4 r n d\left(m^{2}+m\right)\right)}}{2}\right]\right\}
$$

onde $r n d \in(0,1)$ é um número aleatório uniformemente distribuído e $\lceil b\rceil$ é o menor inteiro maior do que $b$.

Passo 4. Faça o crossover de um ponto: $\operatorname{Crossover}\left(r_{i}, r_{j}\right)=\left\{r_{x}, r_{y}\right\}$;

Passo 5. Se $r_{x}$ e $r_{y}$ forem cromossomos viáveis, faça:

$$
\begin{aligned}
& \left\{\begin{array}{l}
r_{t}=r_{x}, \text { se fitness }\left(r_{x}\right) \leq f i t n e s s\left(r_{y}\right) \text { e vá ao Passo } 7 ; \\
r_{t}=r_{y}, \text { caso contrário }
\end{array}\right. \\
& \text { ou então, se apenas um entre } r_{x} \text { e } r_{y} \text { é viável, faça: } \\
& r_{t}=r_{x} \text { ou } r_{y} \text { (viável) e vá ao Passo } 7 ;
\end{aligned}
$$

Passo 6. Faça mutação (no caso de $r_{x}$ e $r_{y}$ serem não viáveis)

- Escolha aleatoriamente $r_{x}$ ou $r_{y}$;

- Faça a mutação no cromossomo escolhido, produzindo $r_{t}$;

Passo 7. Se fitness $\left(r_{t}\right)<$ fitness $\left(r_{m}\right)$, faça:

- Elimine $r_{m}$ (o pior cromossomo) da lista $R$;

- Insira $r_{t}$ na lista $R$, mantendo a ordem crescente dos fitness;

- Faça $k=k+1$ e volte ao Passo 2 .

Considerando-se o aspecto probabilístico dos algoritmos genéticos, foram efetuadas 5 simulações. Em cada uma dessas simulações, a partir da solução fornecida pelo AGPMed, iniciou-se o algoritmo de T\&B, cujos passos estão descritos a seguir, buscando melhorar a solução através da substituição de vértices. Nesta etapa, que define a melhor localização das 12 medianas, não foram consideradas as capacidades de cada mediana (que está representando cada leiturista, neste trabalho).

\section{Algoritmo de Teitz e Bart}

Passo 0. Selecione, aleatoriamente, um conjunto $V_{p} \subset V$, com $\left|V_{p}\right|=p$ para formar uma solução inicial para o problema;

Passo 1. Rotule todos os vértices $v_{i} \in\left\{V-V_{p}\right\}$ como "não analisados"; 
Passo 2. Enquanto existirem vértices não analisados em $\left\{V-V_{p}\right\}$ faça o seguinte: Selecione um vértice não analisado $v_{i} \in\left\{V-V_{p}\right\}$, e calcule a redução $\Delta_{i j}$ do número de transmissão, para todos os vértices $v_{j}$ pertencentes a $V_{p}$, ou seja: $\Delta_{i j}=\sigma\left(V_{p}\right)-\sigma\left(V_{p} \bigcup\left\{v_{i}\right\}-\left\{v_{j}\right\}\right), \forall v_{j} \in V_{p}$.

Faça $\Delta_{i j \_ \text {máx }}=\operatorname{máximo}\left[\Delta_{i j}\right]$, para todo $\Delta_{i j}$ calculado anteriormente. Se $\Delta_{i j \_ \text {máx }}>0$ então:

Faça $V_{p}=\left(V_{p} \bigcup\left\{v_{i}\right\}-\left\{v_{j}\right\}\right)$ e insira $v_{j}$ em $\left\{V-V_{p}\right\}$.

Rotule $v_{j}$ como "analisado".

Caso contrário, continue.

Passo 3. Se, durante a execução do Passo 2, houver alguma modificação no conjunto $V_{p}$, então volte ao Passo 1 e continue a execução do algoritmo. Caso contrário, PARE e apresente o conjunto $V_{p}$ como uma solução aproximada para o problema das $p$-medianas.

Os resultados de algumas simulações executadas até esta etapa (aplicação dos algoritmos AGPMed e T\&B) são apresentados na Tabela 1 a seguir.

\begin{tabular}{c|ccc|cc}
\hline & \multicolumn{3}{|c|}{$\begin{array}{c}\text { Algoritmo Genético } \\
\text { (AGPMed) }\end{array}$} & \multicolumn{2}{c}{$\begin{array}{c}\text { Algoritmo de Teitz e Bart } \\
\text { (T\&B) }\end{array}$} \\
\hline Simulação & $\begin{array}{c}\text { Número } \\
\text { Iterações }\end{array}$ & $\begin{array}{c}\text { Tamanho } \\
\text { População }\end{array}$ & $\begin{array}{c}\text { Valor } \\
\text { Transmissão } \\
\text { (metros) }\end{array}$ & $\begin{array}{c}\text { Número } \\
\text { Iterações }\end{array}$ & $\begin{array}{c}\text { Valor } \\
\text { Transmissão } \\
\text { (metros) }\end{array}$ \\
\hline 1 & 1000 & 200 & 201.569 & 17 & 185.152 \\
2 & 1000 & 150 & 202.306 & 14 & 184.663 \\
3 & 1000 & 75 & 203.176 & 18 & 184.920 \\
4 & 1000 & 200 & 202.946 & 12 & 187.156 \\
5 & 1000 & 100 & 213.958 & 15 & 186.164 \\
\hline
\end{tabular}

Tabela 1: Resultados numéricos das simulações para o problema das 12-medianas.

Ficaram assim definidos 12 pontos, dentre os 774 disponíveis, que representam uma boa aproximação para o problema de localização de 12 medianas do grafo. Estas 12 medianas servirão como "pontos de apoio" para a formação dos clusters de atendimento ( $3^{a}$ etapa).

A $3^{a}$ etapa consta em designar a cada uma destas 12 medianas, os demais nós $(774-12=762$ nós $)$, fazendo com que cada um atenda, aproximadamente, a mesma quantidade de clientes. Nesta etapa, considerou-se a restrição de capacidade associada a cada mediana. Para tanto, utilizou-se o algoritmo de designação proposto por Gillett e Johnson (G\&J), com a adaptação que inclui a restrição de capacidade, Bodin et al. [2], que está descrito a seguir.

\section{Algoritmo de Gillett e Johnson Adaptado}

Passo 1. Calcule a distância entre cada nó $i$ ainda não designado, até cada uma das medianas (leituristas), cujos leituristas correspondentes ainda possuem 
"capacidade". A capacidade média de cada leiturista, Cap $=(n-12) / 12$, neste trabalho, é de aproximadamente 65 nós;

Passo 2. Para cada nó $i$ do passo anterior, defina $t_{i}^{1}$ como sendo o depósito mais próximo de $i$ e $t_{i}^{2}$ como sendo o segundo depósito mais próximo de $i$, com distâncias iguais a $c_{i}^{1}$ e $c_{i}^{2}$, respectivamente;

Passo 3. Para todos os nós $i$ dos passos anteriores, calcule a razão $r_{i}=c_{i}^{1} / c_{i}^{2}$. Ordene os nós $i$ de acordo com os valores de $r_{i}$, em ordem crescente. Esta lista determina a ordem em que os nós serão designados a cada uma das medianas: aqueles nós relativamente próximos a uma mediana serão considerados primeiro;

Passo 4. Percorra a lista do passo anterior, designando os nós $i$ às medianas mais próximas, até que a "capacidade" de alguma delas fique esgotada. Neste caso, retire todos os nós $i$ já designados e a mediana (leiturista) com "capacidade" esgotada e volte ao Passo 1.

Através da designação dos pontos a cada uma das 12 medianas, é obtida a formação dos 12 clusters de atendimento, que podem ser visualizados na Figura 1, onde os símbolos maiores indicam as medianas e os menores, os demais pontos de demanda.

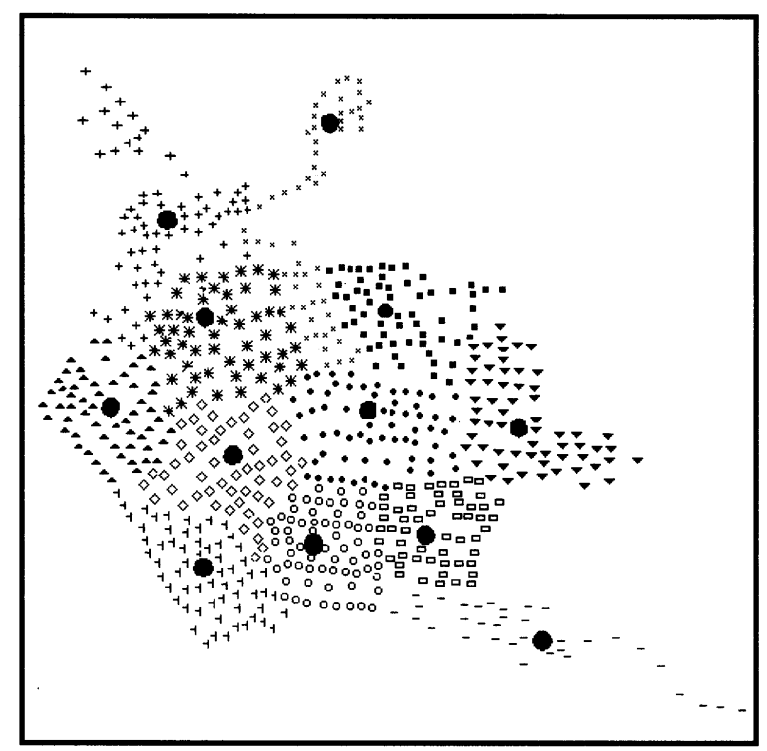

Figura 1: Definição das 12 medianas (pontos maiores) pelos algoritmos AGPMed e $\mathrm{T} \& \mathrm{~B}$ e dos 12 clusters (pontos menores) pelo algoritmo de G\&J.

$\mathrm{Na} 4^{a}$ e última etapa são construídos os roteiros através dos pontos pertencentes a cada um dos clusters a serem percorridos pelos leituristas. O roteamento através dos pontos desses clusters é classificado como um problema de cobertura de arcos, 
onde deve-se determinar um caminho euleriano mínimo através de um grafo tal que, todos os arcos (trechos) sejam atravessados uma única vez.

O primeiro pesquisador a relatar esse problema foi o pesquisador Kwan Mei-ko, Mei-ko [9], onde ele definiu o problema de um carteiro que devia cobrir seu local de trabalho, e retornar ao posto, percorrendo a menor distância de percurso. Por esse motivo, o problema foi denominado Problema do Carteiro Chinês (PCC). O PCC pode ser considerado sobre um grafo orientado ou não, sendo que em ambos os casos existem algoritmos polinomiais para a resolução do problema, Edmonds \& Johnson [5]. A solução do PCC em grafos não-orientados quando o grafo é dito euleriano, reduz-se a determinação de um ciclo de custo mínimo. Um grafo conexo $\mathrm{G}$ é euleriano quando possui um número par de arestas incidentes em cada nó. Caso o grafo não seja euleriano, ou seja, se existirem nós de grau ímpar, será necessário percorrer algumas arestas mais de uma vez para que seja possível a obtenção de tal ciclo.

A formulação matemática do Problema do Carteiro Chinês (PCC), Bodin et al. [2], é apresentada a seguir.

$$
\begin{array}{rlll}
\text { Minimizar } & \sum_{i=1}^{n} \sum_{j=1}^{n} c_{i j} x_{i j} & & (a) \\
\text { sujeito a : } & & \\
& \sum_{j=1}^{n} x_{j i}-\sum_{j=1}^{n} x_{i j}=0, \quad i=1, \ldots, n & (b) \\
& x_{i j}+x_{j i} \geq 1, & \forall(i, j) \in A & (c) \\
& x_{i j} \geq 0 \text { inteiros } & & (d)
\end{array}
$$

onde a função objetivo $(a)$ minimiza o custo total, ou seja, a distância total a ser percorrida. As restrições em $(b)$ garantem a continuidade da rota; as restrições em $(c)$ que nenhuma aresta deixará de ser considerada, e em $(d)$ tem-se que as variáveis do problema são não negativas e inteiras.

O modelo matemático (equações $(a)$ a $(d)$ ) foi desenvolvido para cada um dos 12 clusters separadamente, sendo que a restrição $x_{i j}+x_{j i} \geq 1$ foi substituída por $x_{i j}+x_{j i} \geq 2$ tendo-se em vista que, neste trabalho, a leitura deve ser feita em ambos os lados da rua, já que todos os trechos são produtivos, conforme mencionada no início da Seção 3. Para a resolução destes 12 modelos matemáticos foi utilizado o software LINGO (Language for Interactive General Optimizer) obtendo-se a solução exata para todos os clusters, que totalizou $z=97.839$ metros, como pode-se observar na Tabela 2 a seguir. Nesta tabela, em "Número da Rota" tem-se o número do primeiro ponto a ser transpassado pelo leiturista e em "Distância Percorrida" tem-se a distância discriminada por rota.

\section{Conclusões}

Neste artigo é apresentada uma metodologia, composta de algumas técnicas de Pesquisa Operacional, para a otimização de rotas em um problema de cobertura de arcos. A utilização do AGPMed melhorado com a utilização do algoritmo T\&B 


\begin{tabular}{c|c}
\hline $\begin{array}{c}\text { Número } \\
\text { da } \\
\text { rota }\end{array}$ & $\begin{array}{c}\text { Distância } \\
\text { Percorrida } \\
\text { (metros) }\end{array}$ \\
\hline 64 & 9.073 \\
111 & 8.040 \\
198 & 8.112 \\
272 & 9.204 \\
325 & 7.076 \\
364 & 6.575 \\
\hline
\end{tabular}

\begin{tabular}{c|c}
\multicolumn{2}{c}{ continuação } \\
\hline $\begin{array}{c}\text { Número } \\
\text { da } \\
\text { rota }\end{array}$ & $\begin{array}{c}\text { Distância } \\
\text { Percorrida } \\
\text { (metros) }\end{array}$ \\
\hline 474 & 6.579 \\
541 & 8.445 \\
598 & 8.988 \\
641 & 8.507 \\
679 & 9.185 \\
745 & 8.055 \\
\hline Total & 97.839 \\
\hline
\end{tabular}

Tabela 2: Resultados (distâncias) obtidos pelo Software LINGO.

fornece uma solução satisfatória para o problema das p-medianas (12-medianas, neste trabalho) como pode ser observado nos resultados contidos na Tabela 1. Estas 12 medianas funcionam como "pontos de apoio" para a formação dos clusters, obtidos em seguida através do algoritmo de G\&J adaptado, que apresenta resultados satisfatórios para a designação dos pontos aos respectivos leituristas. Para o roteamento dos pontos pertencentes a cada cluster, o modelo do PCC, utilizado para a obtenção dos arcos a serem transpassados e a respectiva distância total a ser percorrida, mostrou-se eficiente também, cujos resultados estão na Tabela 2.

Comparando-se a solução otimizada (distância total $=97.839$ metros) com a distância percorrida por ocasião da coleta de dados (distância total $=105.793$ metros) tem-se uma melhoria de 7,52\%. Isso representa uma redução média de 7.950 metros no circuito total deste grupo de 774 pontos. Considerando-se que existem outros 6 grupos semelhantes no município de Pato Branco, essa redução pode tornarse bastante significativa, mostrando que a implementação da presente proposta é viável e importante. Com a redução nas distâncias a serem percorridas, a companhia pode diminuir o número de funcionários "em campo" ou, ainda, manter o número e oferecer um serviço de maior qualidade aos seus usuários, já que haverá um tempo adicional para ser gasto nas demais tarefas dos leituristas.

\footnotetext{
Abstract. In this work it is proposed a methodology to get an optimized solution to an arc routing problem using some Operations Research techniques. The methodology presented here is divided into two phases. In the $1^{\text {st }}$ one we use a genetic algorithm applied to the p-median problem, which solution is improved with the Teitz and Bart's classical heuristic algorithm. With the definition of the necessary medians to the problem we define the clusters of demand points to be assigned for each median through Gillett and Johnson's adapted algorithm. In the $2^{\text {nd }}$ phase, it is obtained the routes into each cluster to define the demand points sequence, using the mathematical formulation of the Chinese Postman Problem. The methodology was validated through its use in a case study comparing the optimized solution with the one used at the moment of the data collection.
} 


\section{Referências}

[1] H. Barbosa, "Introdução aos Algoritmos Genéticos", CNMAC, Gramado, RS, 1997.

[2] L.D. Bodin, B.L. Assad e A. Ball, Routing and Scheduling of Vehicles and Crew, The State of the Art. Computers $\&$ Ops. Res., 10 (1983), 69-211.

[3] E.S. Corrêa, M.T.A. Steiner, A.A. Freitas e C. Carnieri, A Genetic Algorithm for solving a Capacitated P-Median Problem. Numerical Algorithms, Kluwer Academic Publishers, 35 (2004), 373-388.

[4] D.M.B. Costa, M.T.A. Steiner, C. Carnieri, L.V.S. Zamboni e A.C.L. da Silva, Técnicas da Pesquisa Operacional na Otimização dos Serviços Postais, Gestão G Produção, 8, No. 1 (2001), 37-55.

[5] J. Edmonds e E. L. J. Matching, Euler tour and the Chinese Postman, Mathematical Programming, 5 (1973), 88-124.

[6] R.W. Eglese e H. Murdock, Routing Road Sweepers in a Rural Area, JORS, 4 (1991), 281-288.

[7] G. Ghiani e G. Improta, An Algorithm for the Hierarchical Chinese Postman Problem, JORS, 26 (2000), 27-32.

[8] D. Goldberg, "Genetic Algorithms in Search, Optimization, and Machine Learning", Addison-Wesley, Menlo Park, CA, 1986.

[9] K. Mei-ko, Graphic Programming using Odd and Even Points, Chinese Mathematics, 1 (1962), 273-277.

[10] E.L.F. Senne e L.A.N. Lorena, Abordagens Complementares para o Problema de P-Medianas, Revista Produção, 13 (2003), 78-87.

[11] A. Smiderle, "Técnicas da Pesquisa Operacional Aplicadas a um Problema de Cobertura de Arcos", Dissertação de Mestrado, PPGMNE, UFPR, PR, 2001.

[12] H.I. Stern e M. Dror, Routing Eletric Meter Readers, Computers 6 Operations Research, 6 (1978), 209-223.

[13] M.B. Teitz e P. Bart, Heuristics Methods for Estimating the Generalized Vertex Median of a Weighted Graph, Operations Research, 16 (1968), 955-961. 